\title{
Unusual Presentation of a Gastrointestinal Tumor in Excluded Stomach - the Need to Think About the Hidden Enemy
}

\author{
Tiago Pereira Guedes ${ }^{1 *}$, Daniela Ferreira ${ }^{1}$, José Ricardo Brandão², José Ramón Vizcaíno², \\ Isabel Pedroto ${ }^{1}$
}

\author{
*Corresponding author: \\ Tiago Pereira Guedes, MD \\ Gastroenterology Department \\ Centro Hospitalar Universitario do Porto \\ Largo do Prof. Abel Salazar, 4099-001 \\ Porto, Portugal \\ Tel: (00351) 916482688 \\ E-mail: tiagoapguedes@gmail.com \\ ORCID ID: 0000-0002-6582-6749
} 1Gastroenterology Department, Centro Hospitalar Universitario do Porto, Portugal
${ }^{2}$ Pathology Department, Centro Hospitalar Universitario do Porto, Portugal

\section{ABSTRACT}

Obesity and its surgical treatment have been increasing in the last decade. Roux-en- $Y$ gastric bypass (RYGB) is one of the most common procedures performed, resulting in an excluded stomach that can be the home of pre- and postoperative pathology. We present a case report of a 52 years-old man with melena and refractory anemia 2 years after RYGB. Although a possible etiology was found through esophagogastroduodenoscopy, the persistence of anemia determined the search for other causes. Additional abdominal CT revealed a tumoral mass in the excluded stomach with subsequent biopsy findings compatible with a gastrointestinal stromal tumor (GIST). Neoadjuvant imatinib therapy and surgery werewas performed confirming the diagnosis and showing GIST ulcerating the mucosa of the excluded stomach. After surgery, imatinib treatment was restarted and maintained so far with no recurrence of anemia. Malignancy of excluded stomach are frequently of epithelial origin and, to our knowledge, GIST occurrence is a very rare finding on an excluded stomach. This highlights the importance of considering the occurrence of hidden pathology in the excluded stomach, as well as the need for a high-qualityhigh quality EGD in pre-bariatric surgery evaluation.

Key words: gastric bypass, gastrointestinal stromal tumor, bariatric surgery, excluded stomach, anemia

\section{INTRODUCTION}

Obesity is becoming increasingly common in the western world with its severe and morbid forms rising in the last decade. Its surgical treatment is a growing reality with sleeve gastrectomy and Roux-en-Y gastric bypass (RYGB) becoming the most common procedures. Both surgical approaches have proven efficacy concerning to weightweigh loss with differences regarding its effects on co-morbidities, with a tailored choice being made according to preexistent medical conditions. Consensus on the necessity of esophagogastroduodenoscopy (EGD) before bariatric surgery is lacking with nearly $10 \%$ of bariatric units considering preoperative EGD unnecessary, whereas $31 \%$ include EGD in their routine preoperative evaluation (1). A recent study showed that 
$23 \%$ (294/1474) of patients had clinically significant findings in preoperative EGD before bariatric surgery supporting it as a necessary diagnostic tool in this context (2). Reports of gastric pathologic findings during bariatric surgery are rare. However, even when lesions are not detected in the preoperative study or surgery, tumors in the excluded stomach may develop and are frequently overlooked. Adenocarcinomas are the most common reported tumors of excluded stomach (3-8). Gastrointestinal stromal tumors (GIST) are commonly located in the stomach and account for $<1 \%$ of all neoplasms of the alimentary tract. Coincidental detection of gastrointestinal tumors during bariatric surgery has been reported to be around $0.8 \%$ (9). When detected before or during surgery, GIST resection is recommended. Diagnostic challenges are due to the nonspecific nature of their symptoms and the difficulty of the endoscopic approach to the gastric remnant. Reported cases of GIST that developed in the excluded stomach after RYGB are very rare (10).

\section{CASE REPORT}

A 52-year-old morbidly obese man was proposed to RYGB in 2015 and, according to our institutional protocol, a high-quality EGD was performed revealing antral erythema with gastric body and antrum biopsies revealing Helicobacter pylori-associatedpylori associated gastritis, without associated pre-malignant conditions. Eradication therapy was prescribed with a subsequent negative urease breath test and RYGB was performed after, without complications. Two years later the patient was admitted to another hospital with complaints of severe asthenia and melena with a oneweek evolution. He was hemodynamically stable and his blood count revealed a normocytic and normochromic anemia with a hemoglobin level of $6 \mathrm{~g} / \mathrm{dL}$. After transfusion of 4 units of red blood cells, he was transferred to our hospital. An EGD revealed an ulcer on the jejunal side of gastro-jejunal anastomosis, involving $50 \%$ of the circumference with hematinic pigment. Treatment with intravenous proton pump inhibitor (PPI) was started and the patient was discharged three days later without new episodes of melena, a stable hemoglobin level of $9.9 \mathrm{~g} / \mathrm{dL}$, and on oral PPI. Despite the absence of visible hematic losses, 20 days after discharge, the hemoglobin value dropped to $8.2 \mathrm{~g} / \mathrm{dL}$ with low ferritin and transferrin saturation. An EGD was repeated demonstrating an improvement with a smaller clear base anastomosis ulcer. Intravenous iron replacement therapy was prescribed without adequate hematological response. An ileocolonoscopy was performed, with no significant findings. At this moment, an abdominal CT scan was performed revealing an expansive and well-defined $13 \times 16 \times 14.6 \mathrm{~cm}$ heterogeneous lesion, with findings suggesting necrotic/ cystic degeneration (fig. 1a). The mass was on the dependence of the excluded gastric wall without a clear cleavage plan with pancreatic body and tail. A CT-guided percutaneous biopsy of the gastric lesion was performed showing cell proliferation of epithelioid phenotype, with slight anisocytosis and anisocariosis, with 8 mitosis figures in the 21 high magnification fields available without evidence of tumor necrosis and with scarce stroma. Immunohistochemical study revealed a negative CAM 5.2 with a strong and generalized expression of CD117/c-kit, weak and focal expressions of smooth muscle actin (S.M.A) and CD34 (fig. 1b-e) and) and preserved cytoplasmic pattern for SDHB. After multidisciplinary discussion, a diagnosis of gastrointestinal stromal tumor (GIST) of gastric origin was assumed and neoadjuvant treatment with imatinib started. In 2018 a gastrectomy of the excluded stomach with distal pancreatectomy and splenectomy was performed with an identifiable gastric fundic lesion invading the retroperitoneum, pancreatic tail, and spleen. The anatomopathological evaluation confirmed the presence of the GIST centered in the muscular layer, with more than $90 \%$ hyalinized stroma and residual spindle cell areas close to the antral mucosa and focally ulcerating it. The tumor expanded through the serosa into the gastrocolic ligament and reaching the pancreatic parenchyma without its invasion orinvading the spleenspleen's. Its anterior surgical margin was also focally intersected. After surgery, imatinib treatment was restarted and maintained so far with no recurrence of anemia. The last CT scan, performed in February 2020 , only only revealed a $22 \mathrm{~mm}$ solid neoplasm in the right kidney meanwhile removed and confirmed to be a primary renal neoplasm. After surgery, imatinib treatment was restarted and maintained so far with no recurrence of anemia. The last CT scan, performed in February 2020, only revealed a $22 \mathrm{~mm}$ solid neoplasm in the right kidney meanwhile removed and confirmed to be a primary renal neoplasm.

\section{DISCUSSION/CONCLUSION}

Malignant tumors developing in the excluded stomach after RYGB are uncommon and most cases reported are of epithelial origin. This case is of particular interest for several reasons. First, the tumor that rapidly developed in the excluded stomach had a mesenchymal origin and, GIST, despite having malig- 

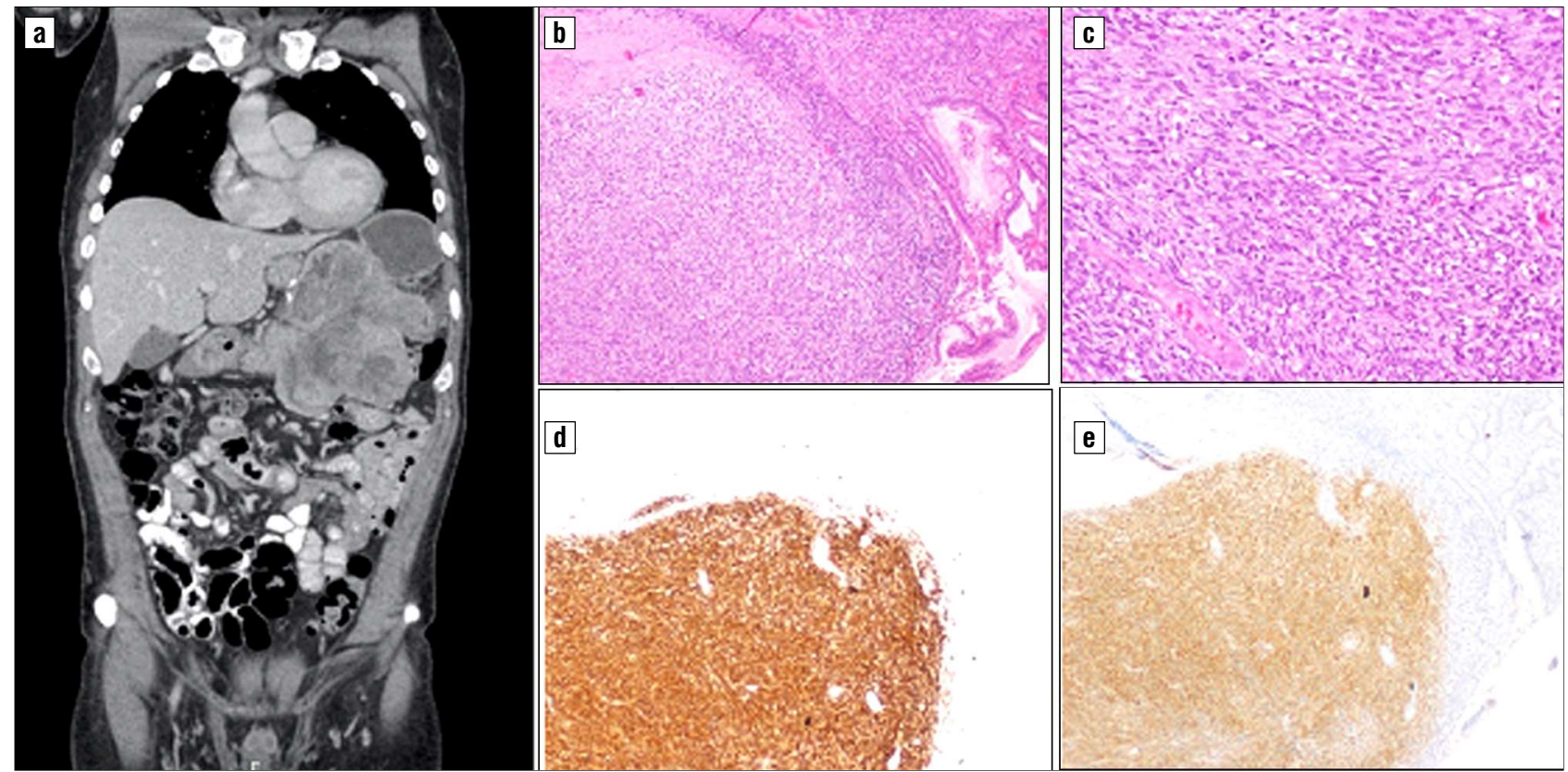

Figure 1 - Abdominal CT with heterogenous lesion of $13 \times 16 \times 14.6 \mathrm{~cm}$, with findings suggesting necrotic/cystic degeneration on the dependence of the excluded gastric wall (a). Fusocellular gastrointestinal stromal tumor in relation to gastric mucosa (H\&E, 40x) (b) and fusiform cells with paranuclear vacuoles and mitotic figures (H\&E, 100x) (c). Cytoplasmic and membrane immunoreactivity do CD117 (c-KIT) (40x) (d) and membrane immunoreactivity to Anoctamin-1 (DOG1) (40x) (e)

nant potential, generally show a more indolent growth. In this case, presurgical EGD and surgical exploration did not detect any changes suggestive of a subepithelial tumor with endo or extra-luminal growth, respectively. This suggests a rapid growth of the tumor after surgery, becoming symptomatic and reaching $14 \mathrm{~cm}$ only 18 months after surgery. Second, the patient presented with melena and EGD revealed an ulcer in the gastrojejunal anastomosis with hematinic pigment, which could justify it. Additionally, after starting treatment with a PPI no hematic losses were seen, which favors it as a cause of melena. However, due to the persistent drop in hemoglobin levels despite endoscopic improvement of the ulcer, another cause for hemorrhage was suspected. The possibility of an iron absorption deficit secondary to hypochlorhydria was raised but did not justify the inadequate response to parenteral iron supplementation. After ileocolonoscopy capsule enteroscopy was considered after ileocolonoscopy, however, bearing in mind the surgical rearrangement, it wouldn't permit the exclusion of lesions in the remnant stomach, so an abdominal CT was done allowing the diagnosis described. The biopsy of the lesion confirmed the diagnosis of GIST. Due to contact of the lesion with the pancreas and spleen, neoadjuvant therapy with imatinib was decided to increase the chances of RO surgery with organ preservation. Interestingly, the result of the surgical specimen revealed that although the GIST originated in the 4th layer of the gastric fundus with extra-luminal growth, it also invaded the gastric antrum in-depthin depth with mucosa ulceration, representing the probable origin of the low-output hemorrhage causing worsening anemia and inadequate response to parenteral iron. With this case, we intend to highlight that in patients submitted to bariatric surgery with persistent iron-deficient anemia not justified by the EGD and colonoscopy findings, the possibility of malignant pathology of the excluded stomach should be kept in mind. The diagnostic difficulties are further compounded by the fact that these patients may also have iron malabsorption from the diet and hypochlorhydria. However, a sub-optimal hematological response to parenteral iron replacement should alert to the presence of digestive losses. Despite the rarity of malignant lesions in the excluded stomach, the delay in diagnosis can have devastating consequences. The endoscopic access to the excluded stomach is possible but challenging. So, from our point of view, in this subgroup of patients, it is advantageous to perform an abdominal image exam such as CT (ideally with enterographyenterographic protocol) to rule out expansive lesions originating in the excluded stomach and/or in the remainder gastrointestinal tract. Also, in this subgroup of patients, abdominal CT should precede capsule enteroscopy that does not allow the evaluation of the excluded stomach and may identify 
common positive findings, such as angiodysplasia, leading to a delay in the diagnosis of malignant pathology of the excluded stomach.

\section{Conflict of interest}

The authors have no funding or conflicts of interest to declare.

\section{Author contributions}

TPG and DF equally contributed to the designing and writing of this manuscript. All authors critically revised the manuscript, approved the final version to be published, and agree to be accountable for all aspects of the work.

\section{Ethical Statement}

The case study was done by following the ethical norms of scientific research and the principles anonymity a confidentiality.

\section{REFERENCES}

1. Zanotti D, Elkalaawy M, Hashemi M, Jenkinson A, Adamo M. Current
Status of Preoperative Oesophago-Gastro-Duodenoscopy (OGD) in Bariatric NHS Units - a BOMSS Survey. Obes Surg. 2016;26(9): 2257-62.

2. Saarinen $T$, Kettunen $U$, Pietiläinen $K H$, Juuti $A$. Is preoperative gastroscopy necessary before sleeve gastrectomy and Roux-en-Y gastric bypass? Surg Obes Relat Dis. 2018;14(6):757-62.

3. Raijman I, Vance Strother S, Donegan WL. Gastric cancer after gastric bypass for obesity case report. J Clin Gastroenterol. 1991; 13(2):191-4.

4. Watkins BJ, Blackmun S, Kuehner ME. Gastric adenocarcinoma after Roux-en-Y gastric bypass: access and evaluation of excluded stomach. Surg Obes Relat Dis. 2007;3(6):644-7.

5. Lord R V, Edwards PD, Coleman MJ. Gastric cancer in the bypassed segment after operation for morbid obesity. ANZ J Surg. 1997;67(8): 580-2.

6. Khitin L, Roses RE, Birkett DH. Cancer in the gastric remnant after gastric bypass. Curr Surg. 2003;60(5):521-3.

7. Corsini D, Simoneti C, Moreira G, Lima S, Garrido A. Cancer in the Excluded Stomach 4 Years after Gastric Bypass. Obes Surg. 2006; 16(7):932-4.

8. Harper JL, Beech D, Tichansky DS, Madan AK. Cancer in the bypassed stomach presenting early after gastric bypass. Obes Surg. 2007;17(9):1268-71.

9. Lyros O, Moulla Y, Mehdorn M, Schierle K, Sucher R, Dietrich A. Coincidental Detection of Gastrointestinal Stromal Tumors During Laparoscopic Bariatric Procedures-Data and Treatment Strategy of a German Reference Center. Obes Surg. 2019;29(6):1858-66.

10. Abellán I, Ruíz de Angulo D, Parrilla P. Incidental gastric gastrointestinal stromal tumor (GIST) in the excluded stomach after Rouxen-Y gastric bypass: A case report and review of the literature. Surg Obes Relat Dis. 2014;10(1):e13-4. 